\section{Getting the most out of your laparoscopic kit: canine}

\section{Philip Lhermette}

Purchasing laparoscopy equipment is a significant expense of around $£ 20,000$. Whilst a good return on this investment can be achieved doing bitch spays alone, the wide variety of procedures that can be performed minimally invasively increases profitability and benefits patients. Laparoscopic bitch spays provide excellent training for the surgeon in instrument/tissue handling and approach to the abdomen. These transferable skills can be applied to other procedures. It may take 60-80 procedures to feel really confident due to the wide variation in the size of patients, but once competent the advantages of a minimally invasive approach are significant.

A wide range of procedures can be performed with very little additional instrumentation, including rhinoscopy, urethrocystoscopy, fistuloscopy, video-otoscopy, thoracoscopy and arthroscopy - further improving practice profitability.

So what other procedures can be done in the abdomen minimally invasively?

\author{
Exploratory laparoscopy \\ Liver/kidney/pancreatic/splenic/lymph node biopsy \\ Laparoscopic-assisted splenectomy \\ Full-thickness intestinal biopsy \\ Ovariectomy/ovariohysterectomy \\ Cystic endometritis/early pyometra \\ Cryptorchidectomy \\ Gastropexy \\ Laparoscopic-assisted cystoscopy \\ Intestinal/gastric foreign body removal \\ - Examination of the gall bladder and biliary tree - \\ cholecystectomy/cholecystocentesis \\ Vasopexy/colposuspension \\ Inguinal hernia repair \\ - Intra-abdominal neoplasia staging/resection \\ - Investigation of intra-abdominal trauma \\ - Adrenalectomy \\ - Extrahepatic shunt attenuation \\ - Nephrectomy \\ - Other operative surgery.
}

\section{ORGAN BIOPSY}

Commonly performed biopsy procedures include liver, pancreatic and full-thickness bowel biopsies. Cholecystocentesis and kidney biopsies can also be performed under laparoscopic guidance and haemostasis can be achieved directly.

\section{GASTROPEXY}

Prophylactic gastropexy has been advocated in at-risk breeds. It is often carried out at the time of other elective surgery, such as laparoscopic ovariectomy, and is easily incorporated into this procedure. Following the spay, an operative port is established approximately $3 \mathrm{~cm}$ caudal to the 13th rib on the right side. The stomach wall is grasped and the port incision is extended to approximately 4 or $5 \mathrm{~cm}$. The stomach wall is elevated into the incision, incised through the serosa and muscular layers and then sutured to the transversus abdominis muscle. The oblique muscles are sutured using simple interrupted sutures. Skin closure is routine.

\section{CRYPTORCHIDECTOMY}

This is simpler, quicker and less traumatic than open surgery! A primary port is introduced near the umbilicus and the testicle can usually be visualized immediately. A secondary port is introduced directly over the testicle which is grasped and then withdrawn from the abdomen together with the cannula. The vas deferens and spermatic vessels are ligated and the testicle removed in routine fashion. Port closure is routine. In most cases the scrotal testicle is also removed, however, if preferred, a vasectomy can be performed on the scrotal testicle intra-abdominally using a vessel sealer/divider.

\section{KEY LEARNING OBJECTIVES}

- Be able to list, describe and compare the advantages and disadvantages of minimally invasive laparoscopic procedures in the dog

- Identify the main laparoscopic procedures that are carried out routinely in the dog using basic laparoscopic equipment

- Describe the procedures for laparoscopic-assisted gastropexy and cryptorchidectomy in the dog

\section{MULTIPLE CHOICE QUESTIONS}

1. Which of the following is NOT a feature of laparoscopic ovariectomy in the dog?
(A) Reduced perioperative pain
(B) Improved visualization of abdominal organs
(C) Reduced operative time
(D) Reduced haemorrhage

2. Which of the following best describes the usual procedure for vasectomy of the remaining testicle following laparoscopic cryptorchidectomy?
(A) The vas deferens on the 'normal' side is isolated through a prescrotal incision and transected
(B) The vas deferens is transected intra- abdominally using a vessel sealer/cutting device
(C) The vas deferens is palpated through the scrotum and exteriorized through a small scrotal incision and then transected
(D) The vas deferens is grasped inside the abdomen, exteriorized through the laparoscopic port and transected 
3. Which of the following best describes the optimal site on the stomach wall that is sutured to the abdominal wall during laparoscopic-assisted gastropexy?

(A) A point directly over the pylorus on the ventral side of the stomach
(B) A point $10 \mathrm{~cm}$ from the pylorus on the greater curvature of the stomach

(C) A point $5 \mathrm{~cm}$ from the pylorus midway between the greater and lesser curvature of the stomach

(D) A point $6 \mathrm{~cm}$ from the pylorus on the lesser curvature of the stomach

\section{Getting the most out of your laparoscopic kit: feline}

\section{Philip Lhermette}

Most practices invest in laparoscopic equipment in order to carry out bitch spays. However the same benefits apply to cats and laparoscopic cat spays can be performed routinely in general practice, not only benefitting our patients but also increasing practice profitability. Indeed cats make ideal laparoscopic patients having a relatively expandable abdomen and less falciform fat.

Most of the procedures that are carried out laparoscopically in dogs can also be done in cats and clients are very open to having minimally invasive procedures rather than open laparotomy, especially for diagnostic procedures such as organ biopsy. The same basic equipment can be used as in canine laparoscopy, although a smaller, 2.7- $\mathrm{mm}$ endoscope is less unwieldy than the more common 5-mm endoscope, and if a lot of feline procedures are undertaken, smaller $3-\mathrm{mm}$ instrumentation can be an advantage. With the addition of very little additional instrumentation a wide range of other procedures can be performed including rhinoscopy, urethrocystoscopy, fistuloscopy, video-otoscopy, and thoracoscopy - further improving the profitability of the equipment.

So what other procedures can be done in the abdomen minimally invasively?

Exploratory laparoscopy
- Liver biopsy
Kidney biopsy
- - Lancreatic biopsy
- Splenic biopsy
- Full-thickness intestinal biopsy
- Ovariectomy/ovariohysterectomy
- Lryptorchidectomy
- Intestinal/gastric foreign body removal
- Examination of the gall bladder and biliary tree
- Intra-abdominal neoplasia staging/resection
- Investigation of intra-abdominal trauma

- Nephrectomy

- Other operative surgery.

\section{ORGAN BIOPSIES}

Commonly performed biopsy procedures include liver, pancreatic and full-thickness bowel biopsies. Cholecystocentesis and kidney biopsies can also be performed under laparoscopic guidance and haemostasis can be achieved effectively under direct visualization.

\section{CRYPTORCHIDECTOMY}

Cryptorchidectomy can also be carried out in the cat in a similar fashion to that described for the dog.

\section{LAPAROSCOPIC-ASSISTED CYSTOSCOPY}

Laparoscopic-assisted cystoscopy is the procedure of choice for male dogs and cats or where large stones are to be removed. Bladder polyps or transitional cell carcinomas may be ablated using a diode laser via this approach. Visualization is greatly improved over traditional open cystotomy and postoperative pain is reduced.

The abdomen is insufflated and a primary camera port is placed at or around the umbilicus. A second port is placed at the midline directly over the apex of the bladder and the bladder wall is grasped and bought up to the abdominal incision where it is sutured to the abdominal wall to form a seal. The endoscope is transferred into an incision made in the bladder wall to inspect the interior of the bladder and instruments can be passed alongside to retrieve stones etc.

At the end of the procedure, the bladder wall is sutured in the normal fashion, the stay sutures are removed and the bladder is returned to the abdomen. The abdomen is fully deflated and all ports and catheters are removed. Closure is routine.

\section{KEY LEARNING OBJECTIVES}

- Be able to list, describe and compare the advantages and disadvantages of minimally invasive laparoscopic procedures in the cat

- Identify the main laparoscopic procedures that are carried out routinely in the cat using basic laparoscopic equipment

- Describe and discuss the procedure for laparoscopic-assisted cystoscopy in the cat 